\title{
The optimize project: beyond first-line therapy in metastatic renal cell carcinoma
}

\author{
Daniel Castellano • Joaquim Bellmunt
}

Published online: 6 July 2012

(C) Springer Science+Business Media, LLC 2012

The treatment of patients with metastatic renal cell carcinoma (mRCC) has changed dramatically over the past 5 years, and a new paradigm has evolved. Angiogenesis in general, and the vascular endothelial growth factor and mammalian target of rapamycin pathways in particular, are validated targets in renal cell carcinoma.

This Supplement Issue examines several interesting topics consequent to the failure of first-line treatment in patients with $\mathrm{mRCC}$, including (1) the reasons why patients fail on first-line treatment with targeted agents; (2) preclinical and clinical rationales for selecting a second- or subsequent-line therapy for these patients; (3) recommendations for managing the main adverse events of targeted therapies, both in normal and special populations; (4) the role of pharmacogenomics in $\mathrm{mRCC}$ to date and whether it is possible to predict an individual patient's outcome and level of toxicity experienced; and lastly (5) how quality of life and supportive care for these patients may be improved.

Currently, tumor progression is most commonly assessed on the basis of the Response Evaluation Criteria in Solid Tumors (RECIST $\mathrm{v} 1.0$ or $\mathrm{v} 1.1$ ). Targeted agents tend to stabilize disease, however, rather than significantly decreasing tumor mass. In the first article, the need for additional methods to assess disease response is discussed. It is also important to evaluate existing prognostic and predictive factors after failure of first-line treatment in order to increase the chances of delivering the most effective and personalized therapy possible.

\footnotetext{
D. Castellano $(\bowtie)$

Medical Oncology Department,

University Hospital 12 de Octubre,

Madrid, Spain

e-mail: cdanicas@hotmail.com

J. Bellmunt

Hospital Universitario del Mar-IMIM,

Barcelona, Spain

e-mail: jbellmunt@parcdesalutmar.cat
}

Despite significant improvements in the first-line treatment of $\mathrm{mRCC}$, therapies rarely produce complete responses, and all patients eventually develop resistance and progress. In addition, some tumors display intrinsic resistance, which further limits therapeutic options. One approach to overcoming tumor resistance is the use of sequential therapy. Unfortunately, knowledge about the mechanisms of action of new therapies, and the intrinsic and extrinsic mechanism of resistance, has not evolved as rapidly as their development and many questions remain unanswered. In the second article, authors review the available evidence from clinical studies on sequential therapy for $\mathrm{mRCC}$, including those that are still in progress. In addition, information on the mechanisms of resistance or tolerance to first-line therapy, recommendations on the main practice guidelines for second-line treatment, potential therapies for third or successive treatment lines, and the reasons why patients who progress may benefit from a change of mechanism of action are also discussed.

In the third article, authors review the toxicity profiles of targeted therapies, and develop important recommendations for treating adverse events to avoid acute, life-threatening situations, dose reductions, interruptions, or early treatment discontinuation. This is essential because the early recognition and management of severe adverse events is one of the most important factors for maintaining the optimum efficacy of sequential therapy.

Pharmacogenomics is the topic of the fourth article, in which the authors evaluate how variation in genetic background affects an individual's response to a specific drug and/or its metabolism. Using knowledge about the genes that produce the enzymes to metabolize a specific drug, a physician may decide to raise or lower the dose, or even change to a different drug. Although few studies have identified biomarkers that predict the response to targeted drugs in the treatment of $\mathrm{mRCC}$, some important associations have been found and are described in this article. Pharmacogenomics holds the potential in the near future to hone 
medical and pharmaceutical tools to produce precision drugs that are more efficient, thus reducing the risk of toxic side effects.

Lastly, the fifth manuscript reviews two key issues in the management of patients with mRCC: the assessment of health-related quality of life (HRQoL) and ways to optimize supportive care. HRQoL during first-line treatment is becoming one of the main factors influencing the choice of subsequent therapies. Consequently, several studies have been conducted to evaluate the impact of approved targeted therapies for mRCC on HRQoL, and this measurement is being introduced with increasing frequency into clinical trial design.

Generating the contents of this supplement issue involved an initial face-to-face meeting in March 2011 between two project coordinators and ten group coordinators who are highly specialized in the treatment of patients with mRCC. These experts defined the topics described above, and nominated the participants of the five working groups. Each working group consisted of two group coordinators and four to six additional members, all of whom are specialized in the management of patients with mRCC. Both group coordinators were responsible for setting deadlines and establishing a dialogue between selected members on the assigned topics in subsequent face-to-face meetings, which took place between May and September 2011. Once each working group had developed the contents of the assigned topics, these were sent to the group coordinators for revision and approval, and ultimately to the project coordinators for final approval. 